\title{
Das Unsichtbare sichtbar machen
}

\author{
Die Networks of Dispossession kartieren türkische Stadtentwicklungsprozesse
}

Yaşar Adnan Adanalı

Nina Gribat
Abb. 1 Projekte der Enteignung (Ausschnitt)

Abb. 2 Partnerschaften der Enteignung (Ausschnitt)

Abb. 3 Enteignete Minderheiten (Ausschnitt)
Dieses Interview mit Yaşar Adnan Adanalı stellt das kollektive Datensammelund Kartierungsprojekt The Networks of Dispossession (Netzwerke der Enteignung) vor, das dieZusammenhänge zwischen Stadtentwicklungsprojekten und der Konzentration von Kapital und Macht in der Türkei untersucht. Wie im Verlauf des Interviews deutlich wird, ist das dem Kartierungsprojekt zugrunde liegende Verständnis von Enteignung sehr breit angelegt. Es bezieht sich auf den Verlust von natürlichen Ressourcen, Stadträumen, Nachbarschaften, Wohnungen und Leben als Folge von Stadtentwicklungsprozessen.

Die Networks of Dispossession umfassen drei Karten (mulksuzlestirme. org): Die erste Karte „Projekte der Enteignung“ (siehe Abb. 1) deckt die Verbindungen zwischen privaten Unternehmen und Regierungsinstitutionen in der Türkei auf; die zweite Karte „Partnerschaften der Enteignung“ (siehe Abb. 2) enthüllt die engen Beziehungen zwischen verschiedenen privatwirtschaftlichen Immobilienentwickler_innen, staatlichen Akteuren und Institutionen sowie Medienunternehmen; die dritte Karte „Enteignete Minderheiten“ (siehe Abb. 3) untersucht, wie im Besitz von Minderheiten befindliche Immobilien, die von staatlichen Organisationen konfisziert wurden, über die Zeit den Besitzer gewechselt haben.

Rechenschaft abzulegen und Prozesse transparent zu machen sind zentrale Anliegen dieses Kartierungsprojekts. Die Daten, die zur Generierung der Karten genutzt wurden, stammen entweder aus öffentlich zugänglichen Quellen, wie zum Beispiel den Webseiten von Unternehmen, der Datenbank der Istanbuler Handelskammer oder dem Amtsblatt für Handelseintragungen, oder aus Sekundärquellen wie Zeitungsartikeln.
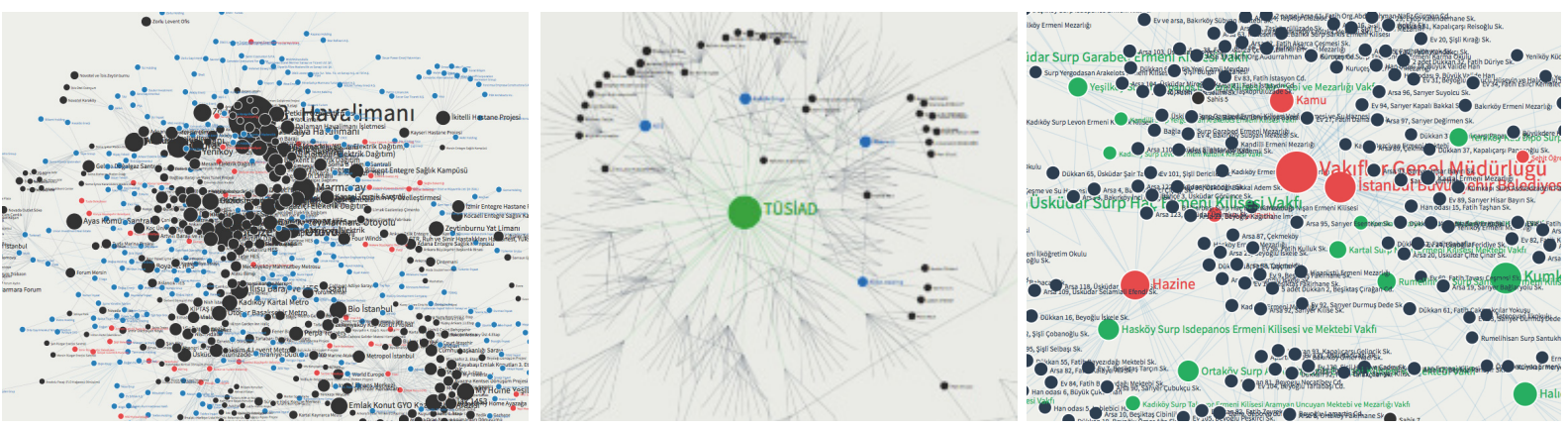
Nina Gribat (NG): Wie entstand die Idee, die Networks of Dispossession zu kartieren?

Yaşar Adnan Adanah (YAA): Am Anfang des Gezi-Protests im Jahr 2013 begann eine Gruppe von Freunden - Burak Arıkan, ein Künstler, ich, ein Stadtforscher und Blogger, ein Paar Journalist_innen, Anwält_innen und Angestellte in Investmentfirmen -, über Stadtentwicklungsfragen zu sprechen, die uns interessierten und zu denen wir schon arbeiteten: Wer sind die Akteure der städtischen Transformation in der Türkei? Wer entwickelt die großen Stadtentwicklungsprojekte, die so viel Unbehagen in der Bevölkerung auslösen? Welche öffentlich-privaten Partnerschaften ermöglichen diese Projekte? Und warum erscheinen diese Projekte so unvermeidlich? Wir entschieden uns, zu diesen Fragen einen Workshop im Gezi-Park zu organisieren. Wir begannen unsere Diskussion mit dem Gezi-Park-Projekt - dem Vorhaben der Regierung, die Taksim-Baracken anstelle des Parks wieder aufzubauen, was auch der Auslöser für die Gezi-Proteste war. Dann setzten wir das Gezi-Park-Projekt in Beziehung zu anderen Stadtentwicklungsprojekten. Nach dem Treffen im Park haben wir uns online organisiert. Zunächst haben wir einen generellen Rahmen für unsere Untersuchung definiert. Alles, was wir dann zu tun hatten, war eine Datenbank zu generieren. Wir haben eine Tabelle angelegt und angefangen, Informationen zu sammeln über verschiedene Stadtentwicklungsprojekte und ihre jeweiligen Entwickler_innen. Dann haben wir angefangen, nach anderen Projekten dieser Entwickler_innen zu suchen.

NG: Was können wir auf der Karte „Projekte der Enteignung“sehen?

$\boldsymbol{Y A A}$ : Da sind zunächst die Stadtentwicklungsprojekte zu sehen, sogenannte Megaprojekte - ,mega' in Bezug auf die riesigen Summen von öffentlichen Investitionen, wie zum Beispiel die 22 Milliarden Euro für den neuen Istanbuler Flughafen (siehe Abb. 4), oder ,mega' in Bezug auf die Auswirkungen auf die Stadt, wie durch die Umwandlung einer Nachbarschaft in eine Shoppingmall, oder ,mega' in Bezug auf die ökologischen Folgen, wie im Fall des Baus einer großen Autobahn in einem Waldgebiet im Norden Istanbuls, oder ,mega im Sinne der riesigen kulturellen und symbolischen Bedeutung, wie bei den Plänen, die größte Moschee des Landes auf dem Çamlıca-Hügel zu bauen, die von überall in der Stadt sichtbar sein wird. In finanzieller Hinsicht wird diese Moschee nur ein Prozent des geplanten Flughafens ausmachen, aber in Bezug auf die symbolischen Auswirkungen wird sie vielleicht wichtiger sein. Je mehr, mega diese Projekte sind, desto größer sind auch die Widersprüche und Konflikte, die sie erzeugen.

Seit die AKP-Regierung 2002 an die Macht kam, ist das Wirtschaftswachstum in der Türkei stark abhängig von der Entwicklung des Bausektors. Die Türkei erfährt zurzeit eine städtische Transformation massiven Ausmaßes. Die erwartete Anzahl von Wohneinheiten, die in der Türkei abgerissen und neu entwickelt werden sollen, liegt bei ca. 7 Millionen, ein wesentlicher Teil davon befindet sich in Istanbul. In vielen Fällen werden ganze Nachbarschaften als (Stadt-)Erneuerungsgebiete ausgewiesen, dann abgerissen und neuentwickelt als bewachte Wohnanlagen (gated communities) oder Einkaufszentren. Ein Großteil des Stadterneuerungsprozesses in der Türkei ist geprägt durch

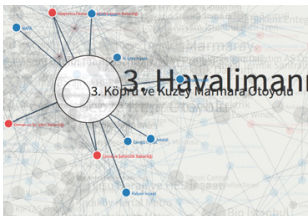

Abb. 4 Das größte Projekt der Karte: der dritte Flughafen Istanbuls (Ausschnitt) 
eine mangelnde Beteiligung der Bewohner_innen an diesen Prozessen und das Versagen von Regierung und Immobilienentwickler_innen, die Belange der Menschen, die in Stadterneuerungsgebieten leben, ins Zentrum zu rücken. Der Erneuerungsprozess in der Türkei ist ein Fall von Enteignung der mittleren bis unteren Mittelschichten durch Erneuerungsprojekte, die für den Profit geplant wurden, fast wie aus dem Lehrbuch.

NG: Welche Stadtentwicklungsprojekte habt ihr kartiert und wo befanden sie sich?

$\boldsymbol{Y A A}$ : Wir haben Stadterneuerungsprojekte in den Außenbezirken und im Stadtzentrum von Istanbul kartiert. Außerdem haben wir kleinmaßstäbliche Wasserkraftwerke in ländlichen Gegenden kartiert, die zwar eine nachhaltige Energiequelle sein sollen, aber doch recht seltsame Entwicklungsprojekte im nördlichen Teil der Türkei sind. Man kann sie überall finden. Sie stellen einen extrem interessanten Fall von Enteignung dar, weil sie so stark von der Regierung als Teil der Umweltpolitik gefördert werden. Diese kleinen Wasserkraftprojekte erstrecken sich entlang der Flussläufe und leiten Wasser vom Flussbett in Röhren um, die im Zickzack den Berg hinunter und durch mehrere Turbinen geführt werden. Während das Wasser auf diesem Weg mehrfach Elektrizität produziert, verliert das Flussbett sein Wasser. Nur sogenanntes Überlebenswasser (survival water) fließt noch im Flussbett, sodass die Natur auch bekommt, was als ihr ,fairer Anteil ${ }^{6}$ verstanden wird. Es gibt Hunderte solcher Projekte und wir haben bemerkt, dass die Gegenden mit besonderem ökologischem Wert, in denen diese Wasserkraftprojekte umgesetzt wurden, sich in den letzten Jahren insgesamt radikal gewandelt haben. Die Dorfbewohner_innen haben ihre Wasserquelle verloren, der Vegetation und den Tieren wurde das Wasser entzogen. Die Firmen, die Wasserkraftwerke entwickeln und betreiben, bekommen auch das Recht, das Wasser, das sie benutzen, zu verkaufen. Am Ende können sie dieses Wasser abfüllen und am Markt verkaufen! Sie haben noch nicht damit angefangen, aber sie könnten. Darüber hinaus können diese Firmen mit den Emissionsrechten am internationalen Markt handeln, weil diese Form der Energieerzeugung erneuerbar sein soll. Das ist eine der problematischsten Formen von scheinbar ,nachhaltiger Entwicklung, die wir haben, und in diesen Dörfern hat sich eine der aktivsten Widerstandsbewegungen dagegen formiert.

Wir haben diese Kombination von Megaprojekten - bestehend aus Stadterneuerungsprojekten, Wasserkraftprojekten und Bergbauprojekten nicht von Anfang an ausgewählt, auch wenn all diese Projekte eine direkte Auswirkung auf die Bevölkerung und die Umwelt und unsere städtischen und dörflichen Commons haben. Wir haben nicht zuerst Kategorien entwickelt und dann nach passenden Projekten gesucht. Wir haben mit dem Gezi-Park-Projekt angefangen und dann nach anderen Projekten gesucht, die von den gleichen Projektentwickler_innen bearbeitet wurden - und so wurden wir auf die Kombination von Projekten, die ich gerade beschrieben habe, aufmerksam. Wir hätten es auch andersherum angehen und mit einer umfassenden Liste von Projekten der Enteignung anfangen können, aber stattdessen haben wir mit der drohenden Enteignung eines Parks durch 
die Überbauung mit einem Megaprojekt, dem Wiederaufbau der TaksimBaracken, angefangen. Von dort hat sich die Karte ausgedehnt, bis sie diese verschiedenen Elemente einschloss.

NG: Warum habt ihr euch entschlossen, Verbindungen von Stadt- und Regionalentwicklungsprojekten zu den Medien zu untersuchen?

YAA: Während der Gezi-Park-Proteste war die Rolle der Medien einer der kritischen Punkte. Es war wirklich schwierig, ausgewogene Nachrichten über die Vorgänge zu bekommen. Ein sehr markantes Beispiel ist, dass CNN Türkei während des Höhepunkts von Gezi einen Dokumentarfilm über Pinguine gezeigt hat, während zum ersten Mal in der türkischen Geschichte etwas Außergewöhnliches in diesem Maßstab geschehen ist! Sie hätten ja noch nicht einmal eine Position beziehen müssen für oder gegen das, was passierte, aber sie hätten wenigstens tun können, was ein Nachrichtenkanal normalerweise tut: von den Ereignissen berichten. Es war so eine einmalige Gelegenheit, die Ereignisse zu verfolgen und von ihnen zu berichten - für jeden Nachrichtenkanal, für jede Zeitschrift: Millionen von Leuten waren auf der Straße! Nachrichtensender lieben solche Ereignisse normalerweise, aber auf CNN Türkei blieb es still. Andere Kanäle wie zum Beispiel NTV berichteten ebenfalls fast gar nicht über Gezi. Schließlich sind die Leute zu den Headquarters dieser Sender gegangen und haben dort protestiert. Die Forderung der Protestierenden war einfach: Wir werden hier nicht weggehen, bis ihr uns auf euren Sendern zeigt. In einer Liveshow sind dann die Fernsehjournalist_innen aus ihren Büros gekommen und haben gesagt: „Vor unseren Büros protestieren Gezi-Demonstranten, weil wir nicht über den Gezi-Park-Protest berichten. "Die Leute haben sich durch die Besetzung des Nachrichtensenders ihr Recht zurück erkämpft, Nachrichten zu empfangen. Während der Gezi-Proteste waren die Medien ein riesiges Problem, weswegen wir entschieden haben, uns auch die Eigentumsverhältnisse im Bereich der Medien als Teil der Networks of Dispossession anzusehen. Wir wollten im Grunde wissen, ob die Firmen, die die großen Stadterneuerungsprojekte bauen, auch die wichtigen Nachrichtenkanäle besitzen.

Uns wurde klar, dass dies der Fall ist (siehe Abb. 5). Zum Beispiel wurde eines der Stadtentwicklungsprojekte, das Tarlabaşı-Erneuerungsprojekt, von der Firma durchgeführt, der auch eines der größten Medienunternehmen gehört, das wiederum vom Schwiegersohn des Premierministers und heutigen Präsidenten Erdoğan geführt wird. Wenn man alle türkischen Medien zusammennimmt und sich ansieht, wie sehr sie in die Networks of Dispossession verflochten sind, dann wird unmittelbar deutlich, dass es eine echte Krise der Demokratie gibt! Deswegen sage ich immer wieder, dass es in der Türkei eine städtische Krise gibt, die Hand in Hand geht mit einer demokratischen Krise.

NG: Darüber hinaus habt ihr die Todesfälle kartiert, die in Verbindung mit den Stadterneuerungsprojekten auftraten. Kannst du ein bisschen darüber erzählen?

$\boldsymbol{Y A A}$ : Wir wollten die Arbeitermorde einbeziehen, um das Argument zu untermauern, dass die geschilderte Entwicklung nicht nur auf Kosten unserer 
Abb. 5 Enge

Verbindungen von

Projektentwicklern und Medienunternehmen (Ausschnitt)
Abb. 6 Der Spine Tower wurde von der Bergbaufirma gebaut, in deren Mine 300 Arbeiter bei einem Unfall ums Leben kamen (Ausschnitt)

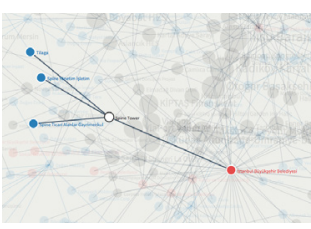

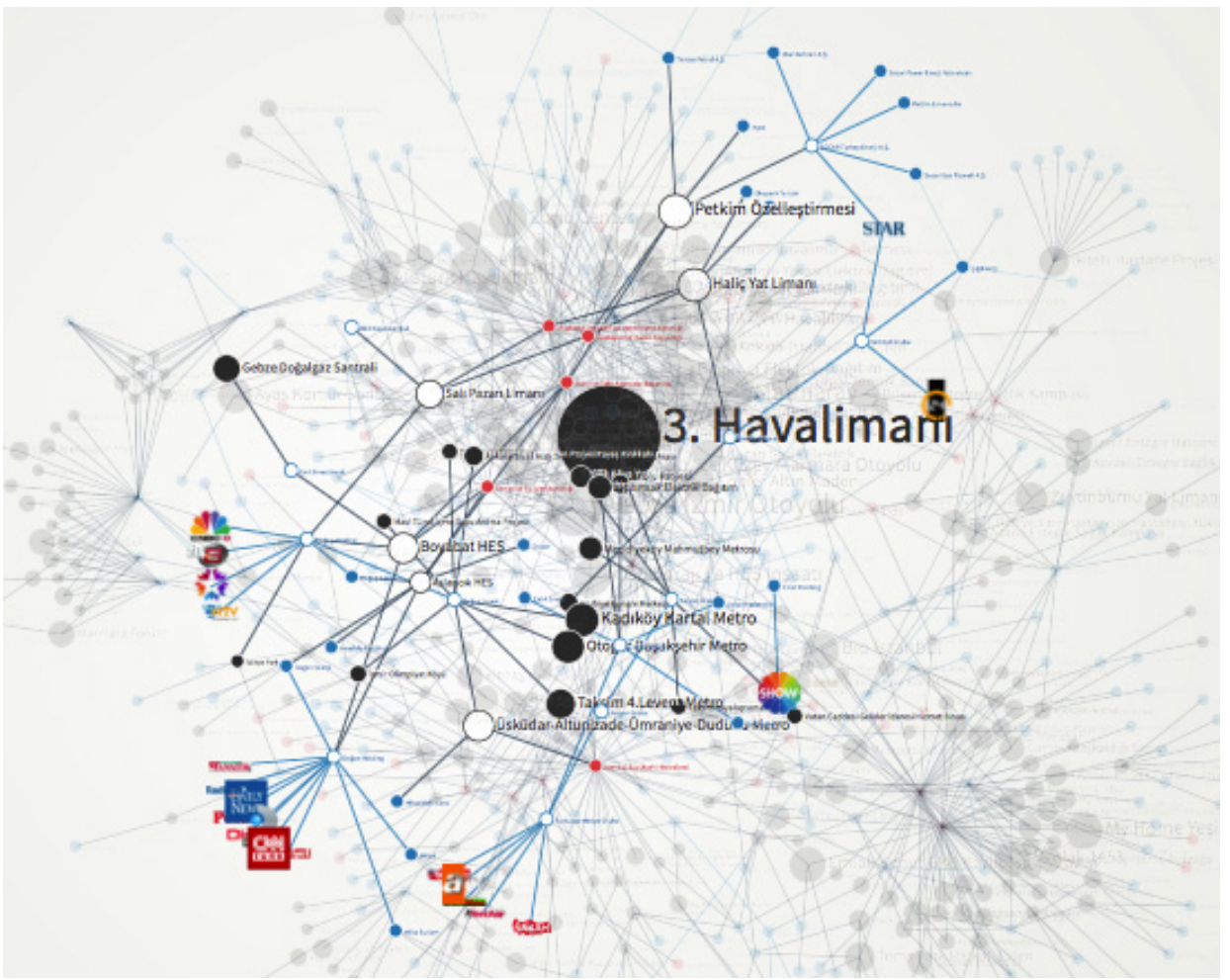

öffentlichen Räume und unserer städtischen und dörflichen Commons vonstattengeht, sondern auch Arbeiter_innen ihr Recht zu leben nimmt. Wir wollten dieses Argument sichtbar machen und haben deswegen die Projekte hervorgehoben, bei denen Arbeiter_innen während des Baus und aufgrund schlechter oder fehlender Sicherheitsbestimmungen ihr Leben verloren haben.

Wie wichtig das war, wurde im Mai 2014 während des Massakers im Bergwerk Soma deutlich. Der Bergbausektor, der in der Türkei zum Großteil als öffentlich-privates Partnerschaftsmodell organisiert ist, beutet die Arbeiter aus. Die Arbeitsbedingungen sind schlecht und es gibt keine staatlichen Sicherheitsbestimmungen. Die Bergbaufirma hat den Mehrwert, den sie von den Minenarbeitern abziehen konnte, in Projekte im Zentrum von Istanbul investiert, etwa in den Bau des höchsten Wolkenkratzers der Türkei. Sie haben sogar behauptet, dieser Wolkenkratzer sei der "sicherste“ in der ganzen Türkei, da sie als Bergbaufirma den besten Zement nutzen könnten. Da sie aber ihre Bergbauarbeiter nicht mit dem Besten von allem versorgt haben, verloren 300 von ihnen ihr Leben im größten Bergbauunfall der letzten Dekade. Ein Immobilienentwicklungsprojekt im Geschäftszentrum von Istanbul das übrigens auch ein Enteignungsprojekt ist, weil es eine sehr umstrittene Baugenehmigung hatte - wurde also ermöglicht durch die Verbindung in eine kleine Bergbaustadt, in der die Firma im Prinzip 300 Leute tötete (Abb. 6). All das war nur möglich, weil die private Bergbaugesellschaft eine Partnerschaft mit dem türkischen Staat schließen konnte. Der Staat gab der Firma eine Garantie, so viel Kohle wie möglich aus dieser Mine zu kaufen, was für die Arbeiter eine Abwärtsspirale in Gang setzte.

NG: Jetzt haben wir die Entwickler_innen, wir haben die Projekte, die Medien und die Arbeiter, die ihre Leben verloren. Du hast angefangen, die 
Verstrickungen des Staates anzudeuten. Kannst du die Rolle des türkischen Staates etwas näher erläutern?

YAA: Zum Beispiel entwickelt die Behörde TOKI (wörtlich: Massenwohnungsbehörde des Premierministers) eine große Anzahl von Immobilienprojekten in Partnerschaft mit privaten Immobilienentwickler_innen, hauptsächlich bewachte Wohnanlagen (gated communities) für die mittlere und obere Mittelschicht in bevorzugten Wohnlagen und sogenannte Sozialwohnungsprojekte für die mittlere bis untere Mittelschicht in den Peripherien der Städte. Diese Projekte werden meist auf öffentlichem Grund und Boden durchgeführt und basieren auf einem Gewinnbeteiligungsmodell. Andere öffentliche Institutionen, wie bestimmte Ministerien oder städtische Verwaltungen, sind in unseren Karten auch prominent sichtbar, weil sie als Verbindungsglieder fungieren. Man kann zum Beispiel ein Cluster von Megainfrastrukturprojekten sehen, das von bestimmten Entwickler_innen geplant wurde, die in sehr enger Verbindung stehen mit der obersten Spitze der Regierung (siehe Abb. 7). Und die Regierungsakteure sind wiederum mit einer Gruppe von großen Bauunternehmen verbunden. Die Typen, die den großen Kuchen entwickeln, haben durch diese Partnerschaften Verbindungen zu den Typen, die die kleineren Kuchen entwickeln. Das zeigt die Karte „Projekte der Enteignung“ (Projects of Dispossession).

Bei der Bekanntmachung der Maps of Dispossession in den sozialen Netzwerken verwendeten wir vor allem den Hashtag \#KentselDönüşümLobisi (\#Stadtentwicklungslobby) - Lobby bezog sich auf den Fakt, dass der Premierminister und seine Ratgeber_innen während der Gezi-Proteste scharf darauf waren, hinter den Protestierenden eine Lobby zu entdecken, wie zum Beispiel die deutsche Flughafenlobby oder die Lobby der US-Interessen. Der Premierminister und seine Berater_innen haben sich diese ganzen verrückten Argumente ausgedacht, was auch zeigt, dass sie keine Absicht hatten zu verstehen, was die Gezi-Protestierenden forderten. Unser Hashtag war eine Art auszudrücken, dass die Verbindungen, die sie bauten, in den Networks of Dispossession die wahre Lobby darstellten - eine Stadtentwicklungslobby.

NG: Wie ist die Karte „Projekte der Enteignung“(Projects of Dispossession) organisiert?

YAA: Sie ist nicht räumlich nach dem Standort der Projekte aufgebaut, sondern wenn man auf die Projekte klickt, kann man Informationen zu ihrem Standort bekommen. Die Kartierung des Netzwerks sollte die öffentlichprivaten Partnerschaften sichtbar machen, auf denen die Projekte beruhen und die sie auch untereinander verbinden. Durch diese Sichtbarkeit wollten wir eine öffentliche Diskussion anstoßen. Wir erhofften uns, dass Leute anfangen würden, die mangelnde Transparenz des Stadtentwicklungsprozesses in der Türkei zu hinterfragen. Unsere grundsätzliche Argumentation war, dass wir durch diese städtischen und ländlichen Entwicklungsprojekte einen Konzentrationsprozess der Macht und insbesondere der Entscheidungsprozesse und eine Konzentration von Kapital sehen könnten. Die folgenden Beispiele des Gezi-Parks und des Bezirks Tarlabaşı zeigen dies: Während wir unseren Park und Tausende Menschen ihre Nachbarschaft verlieren sollten, 
machten andere Leute eine Menge Geld. Auch während die Istanbuler_innen mit dem EMEK-Kino ein denkmalgeschütztes Gebäude verloren, machten andere Leute Geld. Dieser Prozess der Enteignung wurde ermöglicht durch die Konzentration von Kapital und Macht, die sich dadurch weiter verstärkte. Es ist ein Prozess, der zu einer Entdemokratisierung beiträgt. Deswegen argumentieren wir, dass wir uns in einer Krise befinden - einer Krise, die während des Gezi-Park-Protests sehr sichtbar wurde. Wir haben es in der Türkei mit einer Vielzahl ökologischer und demokratischer Krisen zu tun, und unsere Karten zeigen die Verbindungen zwischen diesen scheinbar so unterschiedlichen Krisen auf sehr deutliche Art.

NG: Neben der Projektkarte habt ihr noch ein paar andere Karten produziert. Kannst du mir mehr darüber erzählen?

YAA: Ja, es gibt verschiedene Karten, weil wir durch die Methode der Netzwerkkartierung verschiedene Aspekte herausziehen konnten. Die zweite Karte fokussiert auf die Partnerschaften der Enteignung (Partnerships of Dispossession): Wir haben angefangen mehr zu den involvierten Firmen zu recherchieren - insbesondere den Gremien bzw. Ausschussmitgliedern. Wir sind die Handelszeitschriften und öffentlichen Datensätze durchgegangen, bis wir enthüllen konnten, wie die einzelnen Personen miteinander verbunden sind. Das ist wichtig, weil in vielen Fällen einzelne Individuen in verschiedenen Gremien und Ausschüssen sitzen. Die Entwicklungsfirmen gehören zu verschiedenen Trägergesellschaften, aber am Ende sind sie durch diese Einzelpersonen sehr eng verbunden.

NG: Die Personen sitzen also in verschiedenen Positionen und so wisst ihr, dass das Netzwerk eng gestrickt ist?

$\boldsymbol{Y A A}$ : Ja, genau, das Netzwerk ist eng gestrickt und das hat weitere Folgen, weil diese Firmen alle im gleichen Sektor arbeiten. Theoretisch sollen sie miteinander im Wettbewerb stehen. Nachdem wir kartiert haben, wie eng diese Firmen miteinander verbunden sind, wissen wir, dass es im türkischen Bausektor fast ein Kartell gibt. Die zweite Karte zeigt das.

Die dritte Karte der enteigneten Minderheiten (Dispossessed Minorities) ist eng verbunden mit unserer Entscheidung, die Karten an einem bestimmten Ort auszustellen. Die Networks of Dispossession wurden im September 2013 auf der Istanbuler Biennale ausgestellt. Der Biennale-Ort, an dem wir ausstellen sollten, war eine alte griechische Schule. Diese Schule hat der türkische Staat der griechischen Gemeinschaft vor vielen Jahren weggenommen, ähnlich wie viele andere Immobilien von anderen nichtmuslimischen Gemeinschaften. Diese Gebäude wurden entweder vom türkischen Staat genutzt oder sie wurden privatisiert. Das ist eine andere Art von Enteignung und wir dachten es sei nötig, diese historische Lesart unseren Kartierungen der gegenwärtigen Enteignungsprozesse hinzuzufügen und zu betonen, dass diese aktuellen Partnerschaften zwischen Staat und Kapital in Beziehung stehen zu historischen Prozessen. In der Karte „Enteignete Minderheiten“ kann man bestimmte Gebäude sehen und die Gemeinschaften, denen sie gehörten, wie auch die öffentlichen oder privaten Institutionen, denen diese 
Gebäude heute gehören. Vor ein paar Jahren wurde ein Gesetz erlassen, das es einigen Gemeinschaften ermöglicht, ihre Liegenschaften zurückzufordern. Die Geschichte der Enteignung in der Türkei wird also irgendwie anerkannt. Natürlich ist das sehr schwer umzusetzen, etwa weil sich die Anzahl der Gemeindemitglieder stark reduziert hat, Gemeinden gänzlich verschwunden sind oder manche Gebäude schon abgerissen wurden. Trotzdem hat der Prozess der Rückforderung einen Weg geöffnet, und durch die Karte wollten wir an einem sehr symbolischen Ort unser Statement dazu abgeben.

NG: Wer hat zu der Datenbank, auf der eure Kartierungen basieren, beigetragen? Wie viele Leute wart ihr und woher kamt ihr?

$\boldsymbol{Y A A}$ : Der innere Kreis bestand aus acht bis zehn Leuten, die von Zeit zu Zeit zusammenkamen und ihre Forschungsergebnisse austauschten. Dann hat es sich mehr zerstreut und die Leute haben hauptsächlich an ihren Schreibtischen gearbeitet. Wir haben einige Beiträge aus einem größeren Kreis von vielleicht 20 Leuten erhalten. Nachdem die Karten einen gewissen Stand erreicht hatten, haben wir ein paar Workshops veranstaltet, um Feedback zu bekommen. Dieser erweiterte Kreis hat auch einiges beigetragen. Die Leute kamen aus ganz unterschiedlichen Disziplinen, beteiligt waren Stadtforscher_innen, Anwält_innen, Investor_innen, Künstler_innen, Aktivist_innen und Journalist_innen. Unser gemeinsames Interesse brachte uns von unterschiedlichen Orten aus zusammen. Die Leute haben ihre eigenen Spezialisierungen und ihr Wissen eingebracht. Die Arbeit selbst erforderte keine besonderen Fertigkeiten oder besonderes Wissen - einfach nachzuforschen war schon genug.

NG: Wie funktionierte die Übersetzung der von euch geschaffenen Datenbank in eine Karte?

$\boldsymbol{Y A A}$ : Es handelt sich im Grunde um ganz einfache Visualisierungen. Als Infrastruktur haben wir das Programm Graphcommons verwendet (http:// graphcommons.org), das unser Kollege Burak Arıkan entwickelt hat. Es ist eine Open-Source-Kartierungsplattform, mit der man diese Art von Netzwerken aufbauen kann. Dann haben alle ihre Beiträge hinzugefügt. Wir hatten Journalist_innen im Team, die über die Karten geschrieben haben. Sie waren anonyme Mitglieder unseres Teams, die ihre Beteiligung verdeckt haben, die aber zu dem Projekt beigetragen haben, indem sie darüber berichteten. Wir hatten auch Leute dabei, die in Investmentfirmen arbeiteten. Sie wussten eine Menge über die Stadterneuerungsprojekte. Was diese Freunde beitrugen, kann man nicht „Insiderwissen“ nennen, weil alles auf öffentlichen Informationen basierte. Nach manchen Informationen hätte man einfach intensiver suchen müssen. Für jemanden, der schon Informationen hatte, war der Zugang dazu natürlich viel einfacher. Ich schrieb und war bereit, die Karten öffentlich zu promoten. All diese Elemente haben schließlich zusammengefunden und es hat geklappt...

NG: Ihr habt also die Karten veröffentlicht und verbreitet. Wie wurden sie aufgenommen? Was passierte dann? 
$\boldsymbol{Y A A}$ : Zuerst hat die Istanbuler Biennale geholfen, die Karten bekannter zu machen. Sie fragten uns, ob wir teilnehmen wollten. Ich war schon im Austausch mit dem Biennale-Team, nicht wegen der Karten, sondern wegen diverser anderer Dinge. Unser Künstlerfreund Burak kommunizierte auch mit ihnen. Als sie das Potenzial unserer Arbeit sahen, wollten sie uns eine Plattform bieten. Wir haben dann in unserer Gruppe diskutiert, ob es eine gute Entscheidung wäre, zur Biennale zu gehen, weil es schon ein relativ problematisches Megaevent ist. Wir behielten dabei aber all unsere Freiheit. Und wir zogen keinerlei finanziellen Nutzen aus unserer Biennale-Teilnahme. Wir waren uns über den Inhalt und die Wirkung dieser Arbeit im Klaren. Und wir dachten, wenn wir durch diese Plattform viele Leute erreichen können, könnten wir eine große Wirkung erzielen. Die Biennale wurde einfach ein Podium für das, was wir ohnehin schon taten. Wir haben nichts Neues produziert um der Biennale-Teilnahme willen. Das machte einen großen Unterschied, und deswegen haben wir uns entschieden, es zu tun. Durch die Biennale haben wir eine Menge positiver Rückmeldungen bekommen. Sogar die Financial Times hat über die Karten geschrieben und gesagt, es sei eine bahnbrechende Arbeit. Das war ein gewaltiger Erfolg für uns. Die Financial Times sollte ja immer auf der Seite des Kapitals stehen, hat aber doch unsere Wirkung erkannt. Die türkischen Zeitungen haben die Karten stark beworben. Das ermöglichte es uns, mit verschiedenen Gruppen zu sprechen. Erleichternd hinzukam, dass der Eintritt zur Biennale dieses Mal frei war.

Unsere Karten wurden zunächst als künstlerische Arbeiten gesehen, dann als Forschungsarbeiten, dann als politische Lobbyarbeit, dann als ein Stück Journalismus. Sie sprachen verschiedene Gruppierungen an: künstlerische Gruppierungen, die Mediencommunity, Journalistengruppen, die Bevölkerung, Aktivist_innen und Politiker_innen. Es gab Oppositionsmitglieder, die an den Untersuchungen zu den Korruptionsvorwürfen in Verbindung mit den Projekten, die wir kartiert hatten, beteiligt waren. Sie waren sehr an den Karten interessiert und an der Unterstützung unseres Projekts. Sie sendeten uns sogar noch zusätzliche Informationen und wirkten an der Verbreitung der Karten mit. Das lag natürlich zum Teil an der großen Sichtbarkeit, die wir durch die Biennale hatten. Aber ich glaube immer noch, dass selbst wenn wir nicht an der Biennale teilgenommen hätten, wir schließlich auch viele Leute erreicht hätten, weil der Verlauf der Geschichte die Karten einfach sehr sichtbar und wichtig gemacht hat.

\section{NG: Kannst du etwas über die allgemeine Wirkung der Karten erzählen?}

$\boldsymbol{Y A A}$ : Wie schon gesagt hat unsere Arbeit verschiedene gesellschaftliche Gruppen angesprochen. Darüber hinaus war sie eine Botschaft an manche der Entwickler_innen von Projekten, die schon oder noch nicht kartiert wurden. Die Karten machten ihnen deutlich: „Du kannst kartiert werden.“ Es war auch eine Botschaft an die Architekt_innen dieser Projekte, die selbst im Rampenlicht standen. Ich habe auf diversen Events, bei denen Architekt_innen gesprochen haben, bemerkt, dass sie unsere Kartierungen verbreiten. Architekt_innen, die auf diesem Markt aktiv waren, haben sich auf unsere Karten bezogen und ihr professionelles Umfeld gewarnt: „Ihr könntet auch hier drauf sein - passt auf.“ 
Mit diesem Projekt wollen wir auf die öffentliche Diskussion einwirken und einen neuen, öffentlichen Zugang zu der Art erschließen, wie Stadtentwicklungsprojekte bisher diskutiert wurden. Für uns war es sehr wichtig, dass die Karten auf Daten basieren, auf Verbindungen und Sichtbarkeit, so dass jede_r weiß, dass wir nicht einfach spekulieren und rhetorisch anklagen. Wir können unser Argument auf Daten stützen. In Verbindung mit den Korruptionsanschuldigungen vom 17. Dezember 2013 wurden viele Menschen in Untersuchungshaft genommen, die auch auf unseren Karten auftauchen. Das heißt wohl, dass wir etwas gezeigt haben, das falsch gelaufen ist. Zu dieser Zeit gab es eine öffentliche Diskussion über diese Themen, und wir haben unseren bescheidenen Beitrag dazu geleistet. Unsere Karten haben uns ermöglicht zu argumentieren, dass die Korruptionsvorwürfe sich nicht nur darum drehen, dass Geld von einer Tasche in die andere wandert, sondern dass es sich bei dem, was gerade vor sich geht, um einen größeren Stadtentwicklungsprozess handelt, in dem die breite Öffentlichkeit enteignet wird: Enteignet werden ihre Häuser, ihre Wälder, ihr Wasser.

NG: Wie kommt es, dass ihr die weitreichenden Folgen dieser Enteignungen nicht kartiert habt? Etwa Statements wie: „30o Familien haben ihre Häuser verloren..."

YAA: Wir haben die Quellen mit solchen Informationen verlinkt, dort kann man sich über die Effekte der Enteignungen informieren. Wir wollten nicht zu deskriptiv werden und sagen: „Wegen dieses Projekts..."Wir fanden das nicht nötig. Wir haben nur die Arbeitermorde mit aufgenommen, weil wir es sehr wichtig fanden, diese Aussage zu machen. Zum jetzigen Zeitpunkt haben wir andere Effekte der Enteignungen, wie Zwangsräumungen, Gentrifizierung oder ökologische Schäden, noch nicht kartiert.

NG: Was du über die Aufdeckung der Korruptionsfälle erzählst, hört sich erst mal optimistisch an - gibt es Anzeichen dafür, dass sich nun alles zum Besseren wandelt?

YAA: Naja, das stimmt so nicht ganz. Ein paar Mal wollten uns die ganz groBen Mainstream-Fernsehkanäle einladen, aber dann haben sie immer wieder davon abgesehen. Ich habe mich gefragt, ob sie jemand zurückgehalten hat oder ob sie Selbstzensur betrieben haben. Es ist wichtig zu verstehen, dass es in der Türkei zur Zeit unmöglich ist, einen konstruktiven öffentlichen Dialog zu führen, besonders seit Gezi, weil das Krisenmanagement der Regierung daraus bestand, binäre Gegensätze und Feindschaften zu etablieren, die den Anschein erwecken, als wäre das, was gerade in der Türkei geschieht, eine Art Kalter Krieg.

NG: Ist das deine These oder die These der Regierung?

$\boldsymbol{Y A A}$ : Ich interpretiere die Reaktionen der Regierung als einen internen Kalten Krieg. Sie hat die Protestierenden auch offen als „Plünderer“ bezeichnet und behauptet, diese würden mit ,geheimen internationalen Kräften im Untergrund" zusammenarbeiten. Oder sie bringt Lobbyargumente vor: „Die Banker, die Finanzwelt, die Länder des Westens provozieren diese 
sogenannten Bürger." Solche Verschwörungstheorien und polarisierenden Schwarz-Weiß-Bilder sind in der Türkei weit verbreitet.

NG: Und ihr habt darauf reagiert, indem ihr etwas veröffentlicht habt, das nicht auf Spekulationen basiert, sondern auf Fakten.

$\boldsymbol{Y A A}$ : Ja, wir wollten die Diskussion von diesem Gebiet der starken Worte und der nichtfundierten Behauptungen wegbewegen und auf den Bereich von Daten und Fakten lenken. Zum Beispiel gab es einen Vorfall in den Nachrichten, und plötzlich fing die Regierung an zu behaupten, nackte Protestierende hätten auf eine Kopftuch tragende schwangere Gläubige gepinkelt - mitten am Tag. Das war eines dieser Fantasiebilder, die sie zu erschaffen versuchten, indem sie sagten, die Gezi-Proteste würden von einem Lynchmob getragen. In der Vergangenheit, vor nur ein paar Monaten, hätte man diese Leute noch für bedachte Journalist_innen und Politiker_innen gehalten, auch wenn sie auf der anderen Seite standen. Mit solchen Mechanismen versuchte die Regierung, die Gesellschaft zu spalten. Dabei sieht man auf den Videos ganz deutlich, dass es zwischen der vorbeigehenden Frau und den Leuten fast keinen Kontakt gab - es gab noch nicht einmal viel Protest! All diese riesigen Lügen: Die Kampagne der Regierung baute auf etwas auf, was selbst im ersten Moment unmöglich oder gänzlich verrückt klang. Wie konnte das passieren? Wenn du die Videos siehst, realisierst du, dass etwas völlig anderes passierte! Wir waren in dieser verrückten Situation, in der es sehr schwer war, die Leute zu erreichen - sogar mit Fakten.

NG: Ist die Auswahl der Fakten, die ihr visualisiert, schon eine Art der Interpretation? Eure Karten zeigen manche Verbindungen stärker als andere - wie funktioniert dieser Mechanismus der Visualisierung?

$\boldsymbol{Y A A}$ : Als wir die Karten veröffentlichten, gab es eine Menge Gerede, warum wir dieses oder jenes Projekt nicht aufgenommen hatten - als ob wir versucht hätten, bestimmte Projekte oder Akteure zu verstecken. Unsere Networks of Dispossession sind weiter in Arbeit, sie können wachsen. Wir sammeln immer noch Daten und die zweite Version ist schon auf die doppelte Größe angewachsen.

In Bezug auf die Frage, wie die Darstellung funktioniert: Sie bezieht sich auf die Investitionssumme der Projekte - darauf, wie viel Raum sie in der türkischen Wirtschaft in Bezug auf ihr Projektbudget einnehmen. Wir bilden diesen finanziellen Wert über verschiedene Kreisgrößen ab. So kann man die kleinen und die großen Fische sehen. Man kann unterschiedliche visuelle Lesarten entwickeln. Es kann zum Beispiel eine Menge relativ kleiner Projekte geben, die alle vom gleichen Akteur entwickelt wurden - was heißt das nun am Ende? Oder man kann bestimmten Pfaden folgen, das ergibt auch ganz interessante Lesarten: Ein bestimmtes Projekt kann mit einem anderen verbunden werden, an das wir nicht denken würden, wenn wir die Verbindung nicht sehen würden. Die einfache Lesart funktioniert über die Größe der Projekte, wie sie miteinander verbunden sind und wohin einen die Verbindungen führen. Wenn man die Medien mit reinnimmt und die Verbindungen zu den öffentlichen Institutionen, wird es etwas komplexer. 
Es wäre nicht fair, das als „pure Daten“ und nichts weiter zu bezeichnen. Natürlich gibt es eine Interpretation, aber die bleibt immer offen für weitere Interpretationen. Hineinzuzoomen oder herauszuzoomen ist auch ein Weg, sich die Karten anzusehen. Die Intensität der Verbindungen ist ein weiterer. Und die Art, wie manche Akteure als Verbindungsknoten agieren, eröffnet wiederum neue Interpretationen.

NG: Denkst du, dass soziale Medien wichtig waren für die Wirkung eurer Karten?

YAA: Ja, soziale Medien und normale Medien waren zentral, genau wie unsere Bereitschaft, im Fernsehen, im Radio und in den Nachrichten aufzutreten - all das hat geholfen, die Karten sichtbar zu machen. Und natürlich bietet jede_r beim öffentlichen Teilen eine bestimmte Lesart an, zumindest macht man einen Kommentar. Man zeigt den Leuten eine Richtung, die Karten zu lesen. Wenn es ein Ereignis in Verbindung mit einem bestimmten Projekt oder einem bestimmten Akteur gibt, kannst du in die Karte reinzoomen und das in der Karte zeigen. Damit bietest du zugleich einen Kontext der Enteignung an, wodurch dieses spezielle Ereignis eine viel systematischere Lesart gewinnt.

NG: Yaşar, vielen Dank für das spannende Interview.

Interview, Transkription, redaktionelle Überarbeitung und Übersetzung: Nina Gribat

\section{Beteiligte}

Yaşar Adnan Adanalı ist Stadtforscher, Aktivist und Blogger. Er arbeitet zur Zeit an Projekten, die räumliche Gerechtigkeit und städtische Demokratie fördern.

yasaradanali@gmail.com

Nina Gribat ist Stadt- und Planungsforscherin. Sie arbeitet zur Zeit an international vergleichenden Forschungsprojekten, die sich mit Stadtentwicklungskonflikten, schrumpfenden Städten und den Studienreform/-revolten um 1968 beschäftigen.

nina.gribat@tu-berlin.de 